\title{
Influenza outbreaks in nursing homes with high vaccination coverage in Navarre, Spain, 2011/12
}

J Castilla (jcastilc@navarra.es) ${ }^{1,2}$, F Cía 3 , J Zubicoa ${ }^{3}$, G Reina ${ }^{4}$, V Martínez-Artola5 , C Ezpeleta

1. Instituto de Salud Pública de Navarra, Pamplona, Spain

2. CIBER Epidemiología y Salud Pública, Spain

3. Red de Médicos Centinela de Gripe de Navarra, Pamplona, Spain

4. Clínica Universidad de Navarra, Pamplona, Spain

5. Complejo Hospitalario de Navarra, Pamplona, Spain

Citation style for this article:

Castilla J, Cía F, Zubicoa J, Reina G, Martínez-Artola V, Ezpeleta C. Influenza outbreaks in nursing homes with high vaccination coverage in Navarre, Spain, 2011/12. Euro Surveill. 2012;17(14):pii=20141. Available online: http://www.eurosurveillance.org/ViewArticle.aspx?Articleld=20141

In the 2011/12 season, three influenza outbreaks were studied in nursing homes with high vaccination coverage in Navarre, Spain. Attack rates ranged from $2.9 \%$ to $67 \%$. Influenza $A /$ Stockholm $/ 18 / 2011\left(\mathrm{H}_{3} \mathrm{~N}_{2}\right)$ virus strain was isolated from the three outbreaks. Vaccination should be complemented with other hygiene measures in nursing homes. Early detection of influenza outbreaks in nursing homes can aid in their control.

Four influenza-like illness (ILI) outbreaks have been detected in nursing homes in Navarre, Spain, during the 2011/12 wave of seasonal influenza. Three of these outbreaks were reported rapidly after the detection of the first cases. These outbreaks were investigated by the epidemiological surveillance unit in the region in order to identify the causes, to implement control measures and to give recommendations for preventing outbreaks in other nursing homes.

\section{Background}

People living in nursing homes are more vulnerable to influenza infection due to their advanced age, the presence of major chronic diseases, and to the fact that they live together in close vicinity. Accordingly, there is broad consensus on the advisability of annual influenza vaccination for persons living in such conditions [1-3]. Routine vaccination of residents and caregivers can keep these homes from being affected by waves of seasonal influenza. However, in some seasons, when vaccine effectiveness is low because of the mismatch with the circulating virus, this measure is not enough, and more or less extensive outbreaks can occur [4-6].

\section{Investigation of the outbreaks}

The three early detected outbreaks were studied by gathering information directly from the physicians who attended the cases and analysing the information from individual case reports of ILI. Following the European Union case definition [7] ILI was defined as the sudden onset of any general symptom (fever or feverishness, headache or myalgia) in addition to any respiratory symptom (cough, sore throat or shortness of breath). Vaccination data were obtained from clinical records in the nursing homes and were validated by the regional vaccination registry.

A number of cases were selected for swabbing from each outbreak according to different criteria: a random sample of ILI patients, hospitalised patients or all ILI cases (Table). Nasopharyngeal swabs were tested for detection of influenza virus by real time reverse transcription polymerase chain reaction (RT-PCR) and cell culture using a Madin-Darby canine kidney (MDCK) cell line. Isolates were sent to the National Reference Centre, National Center for Microbiology, Majadahonda, Spain, for influenza genotyping.

\section{Description of the 2011/12 influenza season in Navarre}

Influenza surveillance in Navarre is based on automatic reporting of ILI cases from all primary healthcare centers and hospitals. In addition, a sentinel network of 83 primary care physicians and paediatricians take swabs from all their ILI patients for virological surveillance.

In the $2011 / 12$ season, the influenza wave in the general population of Navarre exceeded the epidemic incidence threshold between 9 January and 18 March 2012, with the peak incidence in the third week of February (from 13 to 19 February).

Approximately $59 \%$ of the non-institutionalised population aged 65 and over in Navarre had received the inactivated influenza vaccine. By 1 April, the cumulative ILI attack rate in the non-institutionalised population was $2.1 \%$ overall, and $0.9 \%$ in those aged 65 and over. Preliminary estimates of vaccine effectiveness in preventing confirmed influenza cases are around 50\% [8].

About 58\% (368/640) of the throat swabs from patients in the sentinel physician network were positive for influenza by culture or RT-PCR, with a clear 
predominance of influenza $\mathrm{A}\left(\mathrm{H}_{3} \mathrm{~N}_{2}\right)$ (94\%). Fiftysix of these strains have been characterised: 43 were $\mathrm{A} /$ Stockholm/18/2011 $\left(\mathrm{H}_{3} \mathrm{~N}_{2}\right)$ and 13 were $\mathrm{A} /$ lowa/19/2010( $\left.\mathrm{H}_{3} \mathrm{~N}_{2}\right)$.

\section{Description of the outbreaks in nursing homes, Navarre 2011/12}

The three nursing home outbreaks are described in the table. All three nursing homes had carried out an influenza vaccination campaign in October 2011, reaching coverage from $82 \%$ to $97 \%$. Problems concerning the vaccine lot, its conservation or administration were ruled out by consulting the vaccination registry and through discussions with the staff responsible for vaccination.

The influenza outbreaks occurred between week 4 and week 8 of 2012, coinciding with the epidemic wave in the region (Figure).

Influenza virus $\mathrm{A}\left(\mathrm{H}_{3} \mathrm{~N}_{2}\right)$ was identified in all the three outbreaks, and the genotyped strains were characterised as A/Stockholm/18/2011( $\left.\mathrm{H}_{3} \mathrm{~N}_{2}\right)$, coinciding with the strain most frequently found in the non-institutionalised population during this season. The attack rates were much higher in nursing homes (range $2.9 \%$ to $67 \%$ ) than those in the general population aged 65 and over (0.9\%). The attack rates in vaccinated persons ranged between $2.6 \%$ and $66 \%$. The attack rates did not differ significantly between vaccinated and non-vaccinated persons in any of the nursing homes. During the outbreak investigation 57 ILI patients were detected with vaccine failure, 13 of whom had laboratory-confirmed influenza. In vaccinated persons, the time between vaccination and onset of ILI symptoms ranged between 92 and 142 days. Overall, $5 \%$ of the ILI cases (3/63) in the three nursing homes required hospitalisation, and death occurred in $3 \%$ of those affected (2/63), all of them in vaccinated persons with previous major chronic conditions.

\section{Control measures}

As soon as the outbreaks were detected, hygiene measures (respiratory hygiene, hand hygiene, use of face masks) were intensified and cases were isolated to control the spread of the infection. The virus spread quickly in the first nursing home affected, and the intervention occurred when a large proportion of the residents had already become ill. In the other two nursing homes, the measures were applied early, helping to keep the attack rate at a lower level. Although antivirals were available, prophylaxis was not used in any nursing home. An alert was issued at regional and national level and hygiene measures were recommended in order to prevent possible future outbreaks in other nursing homes.

\section{Discussion}

Influenza outbreaks in nursing homes with high vaccination coverage were detected in Navarre in the 2011/12 season. The attack rates did not differ significantly between vaccinated and unvaccinated persons, and 13 vaccine failures were laboratory confirmed. The results may indicate insufficient vaccine effectiveness to contain the spread of the influenza virus in nursing homes during this season. A recent study has reported moderate vaccine effectiveness in the $2011 / 12$ season in Spain and suggests a limited match between vaccine and circulating influenza viruses [8].

To prevent influenza outbreaks in nursing homes, high rates of vaccination coverage should be achieved in residents and caregivers, and contacts of sick visitors and workers with residents should be restricted. Despite these measures outbreaks may occur, therefore epidemiologic and virologic surveillance systems in nursing homes should be instituted to report on influenza circulation in the resident population, and to allow early notification of suspected outbreaks [5-6].

Early identification of outbreaks of airborne diseases facilitates adequate decision making for their control. When an influenza outbreak is suspected in an institutionalised setting, it is recommended to intensify measures to avoid transmission: covering the mouth and nose when sneezing, frequent hand washing or use of alcohol-based hand sanitizers, use of face masks, separation of sick persons from the rest of the residents, reducing visits and reducing staff movement between different areas of the building. Antiviral drug treatment in cases and in persons exposed may also be useful [5-6].

Some limitations should be acknowledged in the study of these outbreaks. Information on ILI cases and vaccination status among nursing homes' workers and frequent visitors was not systematically collected. The design and the size of the study were not adequate to obtain estimates of the vaccine effectiveness. However, the high attack rate in vaccinated persons and the number of vaccine failures suggest reduced vaccine protection in these nursing homes.

In conclusion, influenza vaccination should be complemented with other hygiene measures in institutionalised settings. Early detection of influenza outbreaks in nursing homes can aid in their control.

\section{Acknowledgments}

This work was supported by the Carlos III Institute of Health (GRo9/0028 and PSo9/01179), by the Spanish Ministry of Health (EC11-302), and by the I-MOVE (Influenza Monitoring Vaccine Effectiveness in Europe) programme funded by the European Centre for Disease Prevention and Control (ECDC).

\section{References}

1. Centers for Disease Control and Prevention (CDC). Prevention and control of influenza with vaccines: recommendations of the Advisory Committee on Immunization Practices (ACIP), 2011. MMWR Morb Mortal Wkly Rep. 2011;6o(33):1128-32.

2. Grupo de trabajo de vacunación de adultos de la ponencia de programas y registro de vacunaciones. Vacunación en adultos. 
TABLE

Characteristics of the influenza outbreaks and nursing homes studied, Navarre, Spain, 2012

\begin{tabular}{|c|c|c|c|}
\hline & Nursing home $1^{\mathrm{a}}$ & Nursing home $2^{b}$ & Nursing home $3^{a}$ \\
\hline Resident population (n) & 66 & 22 & 523 \\
\hline Women & 52 & 13 & 329 \\
\hline Mean age (range) & $80.3(42-97)$ & $81.2(59-97)$ & $86.4(62-104)$ \\
\hline $2010 / 11$ influenza vaccine coverage & $97 \%$ & $91 \%$ & $82 \%$ \\
\hline Cases of influenza-like illness (n) & 44 & 4 & 15 \\
\hline Influenza attack rate (total) & $67 \%(44 / 66)$ & $18 \%(4 / 22)$ & $2.9 \%(15 / 523)$ \\
\hline Vaccinated & $66 \%(42 / 64)$ & $20 \%(4 / 20)$ & $2.6 \%(11 / 426)$ \\
\hline Unvaccinated & $100 \%(2 / 2)$ & $0 \%(0 / 2)$ & $4.1 \%(4 / 97)$ \\
\hline $\begin{array}{l}\text { Comparison of attack rates in vaccinated and } \\
\text { unvaccinated persons }{ }^{c}\end{array}$ & $P=0.549$ & $P=0.549$ & $P=0.496$ \\
\hline Epidemic period (2012) & $24 \mathrm{Jan}-1$ Feb & $30 \mathrm{Jan}-6 \mathrm{Feb}$ & $2 \mathrm{Feb}-3$ March \\
\hline $\begin{array}{l}\text { Mean time between vaccination } \\
\text { and symptom onset (days) }\end{array}$ & 99 & 103 & 127 \\
\hline Swabbing criteria & Random sampling of cases & Hospitalised case & All cases \\
\hline Nasopharyngeal swabs (n) & 7 & 1 & 15 \\
\hline Identification of influenza virus & 6 & 1 & 8 \\
\hline Vaccinated/unvaccinated & $6 / 0$ & $1 / 0$ & $6 / 2$ \\
\hline Virus strains & A/Stockholm/18/2011( $\left.\mathrm{H}_{3} \mathrm{~N}_{2}\right)$ & A/Stockholm/18/2011( $\left.\mathrm{H}_{3} \mathrm{~N}_{2}\right)$ & A/Stockholm/18/2011( $\left.\mathrm{H}_{3} \mathrm{~N}_{2}\right)$ \\
\hline Influenza-related hospitalisations (n) & 2 & 1 & 0 \\
\hline Influenza-related deaths (n) & 1 & 1 & 0 \\
\hline
\end{tabular}

a Urban area (city with 200,000 inhabitants approximately).

b Rural area (village with 1,000 inhabitants approximately).

c Two-tailed Fisher's exact test.

\section{FIGURE}

Incidence of influenza-like illness (ILI) cases in the general population and ILI cases in nursing homes, by week, Navarre, Spain, 2011/12

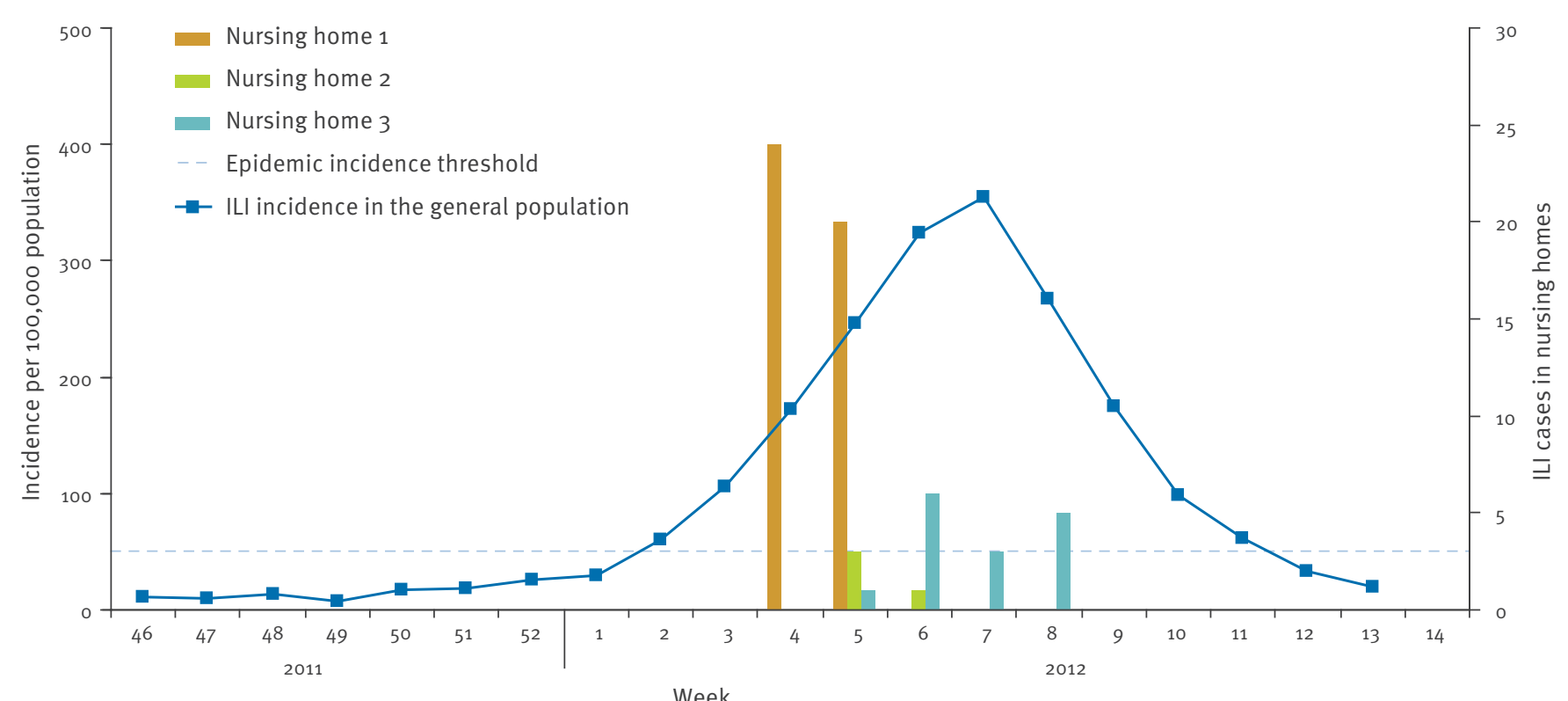


Recomendaciones. [Vaccination in adults. Recommendations]. Madrid: Ministry of Health and Consumer Affairs; 29 Sep 2004. Spanish. Available from: http://www.msssi.gob. es/ciudadanos/proteccionSalud/vacunaciones/docs/ recoVacunasAdultos.pdf

3. Instituto de Salud Pública de Navarra. Protocolo de vacunación antigripal-antineumocócica 2011-2012 [Protocol for influenzapneumococcal vaccination 2011-2012]. Boletín Informativo no 63. Sept 2011. Spanish. Available from: http://www. navarra.es/NR/rdonlyres/AECCD760-AB2A-4841-818AFA53478FD6DC/199452/BOL63GRIPE.pdf

4. Nicholls S, Carroll K, Crofts J, Ben-Eliezer E, Paul J, Zambon $M$, et al. Outbreak of influenza $A\left(\mathrm{H}_{3} \mathrm{~N}_{2}\right)$ in a highly-vaccinated religious community: a retrospective cohort study. Commun Dis Public Health. 2004;7(4):272-7.

5. Monto AS, Rotthoff J, Teich E, Herlocher ML, Truscon R, Yen $\mathrm{HL}$, et al. Detection and control of influenza outbreaks in well-vaccinated nursing home populations. Clin Infect Dis. 2004;39(4):459-64.

6. Castilla J, Red de Médicos Centinela para la Vigilancia de la Gripe en Navarra. Brotes de gripe en población vacunada de residencias geriátricas en la temporada 2004-2005 [Influenza outbreaks in highly-vaccinated older institutionalised people in the 2004/05 season]. Spanish. Med Clin (Barc). 2006;126(1):35-6.

7. European Commission. Commission Decision of 30 April 2009 amending Decision 2002/253/EC laying down case definitions for reporting communicable diseases to the Community network under Decision No 2119/98/EC of the European Parliament and of the Council. Luxembourg: Publications Office of the European Union. 1.5.2009:L 110/58. Available from: http://eur-lex.europa.eu/LexUriServ/LexUriServ.do?uri= 0J:L:2009:110:0058:0059:EN:PDF

8. Jiménez-Jorge S, de Mateo S, Pozo F, Casas I, García Cenoz M, Castilla J, et al. Early estimates of the effectiveness of the $2011 / 12$ influenza vaccine in the population targeted for vaccination in Spain, 25 December 2011 to 19 February 2012. Euro Surveill. 2012;17(12):pii=20129. Available from: http:// www.eurosurveillance.org/ViewArticle.aspx?Articleld =20129 